\title{
Aromatherapy Versus Pharmaceutical Interventions for Dementia Related Behaviors Translating \& Integrating Scholarship Practicum
}

\author{
Vanessa Veit
}

Massachusetts College of Pharmacology and Health Sciences, USA

\author{
Corresponding author \\ Vanessa Veit RN BSN, Massachusetts College of Pharmacology and \\ Health Sciences, USA, E-mail: vlvmvc1@aol.com.
}

Submitted: 26 Oct 2017; Accepted: 02 Nov 2017; Published: 31 Jan 2018

\begin{abstract}
The elderly population is expected to double in the next thirty years increasing the number of individuals with the diagnosis of dementia. By proxy, dementia related behaviors such as agitation, anxiety and restless will also increase. Currently, pharmaceutical management of these behaviors include Ativan, Haldol and other psychotropic drugs which have side effects that place individuals at risk for falls and at times even aggravate the behaviors. Although, not widely researched, aromatherapy is a safe alternative to treating Dementia related behaviors. Research shows aromatherapy has a calming and relaxing effect which can be measured physiologically.
\end{abstract}

\section{Introduction}

It is estimated that by 2030 , the number of elderly individuals will increase to nearly 20 percent of the population [1]. In 2014, almost 50 million people were age 65 or older and by the year 2060 this population is expected to increase to nearly 100 million people [2]. With the growing elderly population, the incidences of age related challenges such as dementia and end of life issues will also increase. Currently, half of individuals in an "acute ward" setting have some sort of dementia [3]. Dementia related behaviors, such as verbal and physical aggression, create challenges with the delivery of care which affects both the caregivers and the residents. Persons with dementia and their caregivers are greatly affected by the problematic symptoms of dementia [4]. Concurrently, terminal agitation, a condition with similar features to dementia related agitation, is a phenomenon characterized by aggression and restlessness. The prevalence of terminal agitation is estimated to be experienced in nearly $85 \%$ of end of life situations [5].

In 2012, due to the increasing population of individuals exhibiting agitated behaviors, a bill to amend the Social Security Act had been proposed in an effort to improve the care and treatment of elderly adults. Had it been passed, the bill would have enforced mandatory education and training for prescribing clinicians regarding antipsychotic medications. The proposed bill had been in response to the growing population of individuals with dementia and the increased off- label use of antipsychotics by clinicians, despite the mortality risk black box warning, as a pharmaceutical intervention to agitation and other dementia related behaviors [6]. Although the bill was not passed in Congress, there has been continuous national advocacy regarding the need for education and resources which promote quality care and treatment for individuals with dementia who exhibit agitated behaviors [7].

With the growing elderly population, the health care industry's focus will most likely be geared towards caring for the geriatric population. Consequently, as we approach end of life, subsequent issues related to the aging process will arise. For example, in 2012, sixty percent of individuals age 65 or older faced two or more chronic illnesses [2]. Looking forward, treating chronic illness or an acute crisis will be at the forefront of primary care. Agitation related to dementia and/or terminal illness will need to be managed by the Primary Care Nurse Practitioner.

One major contributor to terminal agitation is the documented over use of opioids and the negative effects these drugs have on the central nervous system [7]. According to the Regenstrief Institute and the Indiana University for Aging Research, the high frequency of agitated- related emergency room visits has created a rise in hospital and Medicare rates. These cost and rate increases have negatively affected the U.S. economy as a whole [8]. Currently, almost $\$ 215$ billion dollars has been spent annually on providing both private care and professional health care for the elderly in the United States. What will it be in 2060 when the elderly population has almost doubled? [9]. There is a need for alternative interventions which are cost effective to address the growing elderly population and with it, the incidence of dementia related behaviors and/or terminal agitation.

\section{Hypothesis/PICOT Question}

Could aroma therapy be a more effective alternative to pharmaceutical interventions in addressing restlessness, agitation and other stress related effects? There are many methodologies utilized when 
addressing agitated behaviors; however, most include pharmaceutical interventions. Conversely, research into the use of complementary therapies in health care is growing. A recent study regarding the effects of Aroma Acupoint therapy on children with behavioral disturbances demonstrated a positive relationship correlation between the non-invasive therapy and calmness. Each child had a different behavioral disturbance which responded positively to the use of certain scented oils [10].

\section{Methodology}

The theoretical framework utilized in this paper is based on Algase, et al. proposed idea that dementia related behavior is an expression of an unmet need. The framework of a need-driven behavior suggests identifying the need can direct the plan of care and offers alternative approaches in the investigation of such needs [11]. The antagonistic physiological effects of aroma therapy can be qualified as providing physiological needs of the body; providing effective and non-invasive interventions for dementia related behavior.

The data collection for the purpose of this literature review consists of queries of key words in the following search data bases: Academic OneFile, CINAL and Medline (OVID) through the Massachusetts College of Pharmacology and Health Sciences online library databases. Rationale for use of these databases is due to the focus of articles from professional and widely respected nursing and allied health journals [12].

\section{Inclusion of Articles}

The literature chosen was queried by the use of key words which included: aromatherapy, agitation, terminal agitation, Ativan, Haldol, lavender, adverse effects and dementia related behaviors with a date range of 2004- 2016. Variety of environmental factors were included: such as long term care and home care environments. As research is limited, the search was further expanded to include CAM therapies and non-pharmaceutical interventions. All of the articles were in English and published in Peer Reviewed Journals.

\section{Exclusion Criteria}

Non-aroma related therapies and non-English written studies were excluded. Any studies including the effects of agitation or aromatherapy on caregivers and/or family members was also excluded. There was no exclusion to age due to lack of research conducted on a specific age group.

There is limited research in to the area of dementia and psychotropic drug effects and use of aroma therapy. A few articles regarding psychotropic drug use were used to identify risk factors and concern for safety as it relates to the elderly. Other articles included evidenced based practice which supports the hypothesis aromatherapy has any kind of physiological effect on the body. Other articles were chosen to support the hypothesis that this effect, if any, was not harmful, but positive in counteracting negative psychological manifestations.

The first priority was to provide supportive data which creates the forum to ask, what does aromatherapy do? The second priority was to develop a strong foundation for how aromatherapy can counteract the psychological manifestations of stress, agitation and body imbalance. The third priority was to emphasize the potentially negative pharmaceutical safety concerns that arise with the use of psychotropic drugs. Fourteen articles where chosen utilizing the inclusion and exclusion criteria. Each article was further prioritized as a primary reference and then categorized based on clinical findings which suggest a correlation between aromatherapy and body or suggests a correlation between psychotropic medications and the body or correlated dementia related behaviors with physical discomfort. Good studies were relevant to the topic, conducted within the last 10 years and conveyed a high level of reliability.

\section{Critique of Articles}

Chamine and Oken, conducted a quantitative study regarding the effects of aromatherapy on cognitive and physiological functioning within a stressed induced environment. Ninety-two individuals were randomly selected and placed into three aromatherapy groups which included lavender, a coconut, and a placebo (water) group. Cognitive and physical assessments were performed both before (for baseline) and after exposure to a stress battery [13]. Inclusion criteria included ages 50-85, no current medications and nonsmokers. The tools utilized for measurement were a self-measuring questionnaire, the Wechler Adult Intelligence Scale, the BioSemi Active Two electroencephalography test to record respirations and saliva sampling for specific enzyme assays. The findings revealed a correlation between the lavender and the coconut oil and decreased respirations after exposure and post the stress battery. The respirations remained consistent with the placebo (water) group. Working memory testing revealed a $15 \%$ increase in scores for working memory with the lavender over the other groups [13]. The study concluded there was a positive correlation between lavender and working memory and a decrease in stress related physiological findings with both aromas in comparison to the placebo group.

Testad et al. conducted a qualitative study which tested the correlation between psychotropic drug use and severity of agitated behavior. Data was collected from 4 nursing homes from Norway, Austria and England regarding the percentage of psychotropic drug use and the prevalence of agitated behavior in 590 individuals with a known diagnosis of dementia. Inclusion criteria consisted of the severity of dementia based on Functional Assessment Staging Test, Global deterioration scale and clinical dementia rating scales- all very wellknown and respected tools of measurement of dementia. Agitation was measured utilizing the Cohen-Mansfield Agitation Inventory and consisted of 4 categories including physically aggressive behavior, physically non-aggressive behavior, verbally aggressive behavior and hiding/hording behavior [14]. All data collected was analyzed using ANOVA and chi square tests. The study concluded that there was no relationship between the use of antipsychotic drugs and the severity of agitation. In fact, total scores for agitation were less in Norway; this country notably usees less psychotropic drugs compared to Austria and England. The study did conclude there to be a correlation between staff to patient ratio and severity of agitation. Norway had a lower staff to patient ratio (more staff) then the two other countries, less agitation and less use of psychotropic drugs. Limitations affecting the reliability of the study included cross cultural language barriers with measurement tools, and the number of residents participating in the study as it varied from country to country. Austria had the lowest number of residents. Also, variations in sex and age were not considered during the study. Although, more research is needed, this study supports the hypothesis that the prevalence of psychotropic drug use and severity of agitation are not essentially correlated.

Yang, et al. Conducted a quantitative, quasi -experimental study which investigated the potential effects of aroma-acupressure and 
aromatherapy as an intervention for agitated behaviors. Two hundred and seventy-six residents diagnosed with dementia from 3 different retirement homes were selected to participate in the study. Inclusion criteria consisted of a score of 35 or higher utilizing the Mansfield Agitation Inventory. The study excluded any individual with skin issues, and any individual that was hospitalized. Three groups were created: a control group, an aroma-acupressure group, and an aromatherapy group. Baseline electrocardiograms were obtained for each participant. The aroma-acupressure and aromatherapy group participants received a 5-day/week therapy treatments according to the labeled group for a month; the control group received no therapy. Each week a pretest and a posttest electrocardiogram (EKG) and a Chinese version of a CMAI questionnaire of 29 problem behaviors were obtained after receiving treatment [15]. And the EKG results were analyzed. The study revealed a progressive decrease in CMAI scores for the aroma-acupressure and aromatherapy group over the 4 weeks; no change was noted in the control group. Low and high $(\mathrm{LF} / \mathrm{HF})$ frequency values from the EKGs which represent parasympathetic and sympathetic nervous system activity were analyzed. The LF/HF ratios remained stabilized in the aromaacupressure and aromatherapy groups with less notable sympathetic activity noted in comparison the control group [15]. The study is limited due to the following reasons: reliability is low due to the lack of standardization of the CMAI version questionnaire and this was not a blinded study. However, the findings do support evidence of the potential beneficial effects of aroma-based therapies as interventions for negative-dementia related behavior as well as the need for further research.

Nobay, et al. is a quantitative, randomized double blind study which compared the efficacy between Midazolam, Ativan and Haloperidol in controlling agitated behavior by sedation. The study included 111 severely combative and agitated patients of an academically affiliated teaching emergency room department over the course of 2 years. Exclusion criteria included: pregnant women, individuals with known allergies to any of the drugs, bradycardic or tachycardic individuals and individuals under the age of 18. The patient was considered sedated when agitated behavior ceased and one to one supervision was no longer needed [16]. The data collected was imputed and analyzed utilizing the Stat View 5.0 and then compared via the chi-square test. The study revealed there to be less time to sedate and arousal with midazolam. Time to sedation was 32 minutes with Ativan and 28 minutes with Haldol; arousal mean time was 217 minutes for Ativan and 81.9 minutes for Haldol respectively [16]. During the study two patients suffered severe adverse reactions to Haldol. The biggest limitation to this study was any individual who did not become sedated or displayed increased agitated behaviors was excluded from the data and considered sedation failures [16]. The exclusion of such a necessary variable when considering the potential side effects of these drugs renders the study's findings to be skewed and suggestive at best.

Curcani \& Tan, conducted a qualitative quasi-experimental study which tested the effect of aromatherapy on pruritus observed in hemodialysis patients. Between January 2011 and April 2012 data was obtained from questionnaires answered by 134 patients receiving hemodialysis in hospitals located in Turkey. Inclusion criteria included: history of pruritus after receiving treatment within the last 2 weeks, no use of anti-pruritus medication before and during the sampling and no other dermatological problems [17]. The pruritus of each individual was then graded by severity. Two groups were formed, a control group and an experimental group. The control group received conventional nursing care and the experimental group received aromatherapy with a mixture of tea tree, lavender, almond and jojoba oils for six continuous weeks. The data collected was then entered into computers and analyzed via SPSS version 15.00 with Chi-square and t-test analysis. The study revealed a positive correlation between aromatherapy and a lessened severity of pruritus. This study also provided additional support on how aromatherapy affects the body in physiologically calming way.

Pelletier and Landreville, conducted a quantitative cross-sectional study which defined negative behaviors associated with dementia and discusses the correlation between physical discomfort and the expression of those negative behaviors. The article concludes there is strong evidence of a relationship between discomfort caused by an unmet need and agitation in individuals with dementia. The reliability and validity of the article is profound as it clearly defines negative behaviors, utilizes well-respected measuring tools for behaviors, and take in to account the degree of dementia. It further narrows the subject demographics by age, and residence location consistency for at least 3 months. Thirteen nurses with similar backgrounds and experiences chose 49 residents from 3 different nursing homes to participate. However, a large variable unaccounted for in the study was specific to dementia related professional training and identification of nursing home location and specialty. The article did not specify whether these nursing homes were Dementia specific or not. Furthermore, the study included individuals already receiving analgesics with no comparison group without medication. Therefore, the study did not support the hypothesis that greater comfort equals less agitation [18].

According to $\mathrm{Ou}, \mathrm{Lee}, \mathrm{Li} \& \mathrm{Wu}$, aromatherapy can be used as an alternative to pharmaceutical medication in the treatment of neck pain. In this qualitative study, there were two groups, a control group and an experimental group of sixty individuals who suffered from neck pain. The inclusion criteria included ages 20-65 who had received no other treatment for neck pain in the past month. Exclusion criteria included spinal injuries or herniated discs, allergy to essential oils, spinal lesions, neck surgery or a fracture in the past 6 months. For four weeks the control group applied an unscented cream to the neck and the experimental group applied a mixture of marjoram, black pepper, and lavender and peppermint oil to the neck. Researchers followed up with the participants twice a week [19]. An assessment was completed using a visual analogue scale, NDI, pressure pain threshold via meter and neck-joint range with Motion Analysis System both prior to the study, during the study and at completion of the study [19]. All tools of measurement are standardized and reliable. The study depicted a marked improvement with pain in the experimental group in comparison with the control group throughout the course of the study. Moreover, the study hypothesized that aromatherapy stimulates the parasympathetic nervous system facilitating the sensation of pleasure [19].

Finkle, et al. conducted a study of potential fracture risk between zolpidem and benzodiazepines. Prescriptions information was taken from a pharmacy database and cross referenced with hospital records of non-vertebral and hip fractures. This retrospective quantitative cohort study took place over the course of a 10-year- span. Covariates consisted of sex, age and disease [20]. The study revealed that over 42,000 individuals suffered from a non-vertebral fracture, hip fracture and/or dislocation per year within the first 90 days of the 
initial prescription for Ativan [20]. Comparison figures revealed over 10,000 per year for Zolpidem and varied between 16 and 20 thousand for other benzodiazepines respectively. This study illustrates the adverse effects of psychotropic drugs, the high risk for fractures and the consequential medical costs which coincide.

According to Kirland, et al. almost six percent of individuals with dementia who undergo hip surgery die within 30 days of discharge. The quantitative cohort study took place over the course of two years between January 2000 and June 2002. Data was collected from the medical records of consenting patients. The statistical methods used to analyze the data were the Person X squared test and analyses were performed via the SAS version 9.1.3. The study included a total of 485 patients; recorded data included sex, age, Charlson Comorbidity Index score, body mass index (BMI) and admission glucose. Forty out of the 485 patients died within 30 days of discharge. There was no variance with length of stay or glucose value. Lower BMI did show higher risk for mortality. The reliability and validity for this study is high; however, one limitation with this study was limited to one region and one hospital. The study concluded there to be a correlation between advanced age, hip surgery and mortality [21]. Therefore, it can be purposed that because psychotropic medications increase risks for a fall, these medications also increase risk for mortality.

Kimura and Takamatsu, conducted a pilot study to investigate the efficacy of lavender oil on behavior and psychological symptoms of dementia. The study included 20 individuals diagnosed with frontotemperal lobar degeneration (FTLD) over the course of 8 weeks. The inclusion criterion was the diagnosis of FTLD and the mean age was 77. The Japanese version of the Neuropsychiatric Inventory (NPI) was used to assess behavior symptoms of the patients before, during, and after the study. Patients were to receive the lavender oil for 4 continuous weeks and then therapy was discontinued for another 4 weeks for comparison and analysis [22]. Over the course of 4 weeks with lavender therapy, the NPI of the participants dramatically improved and the use of psychotropic drugs decreased. Conversely, the NPI score declined over the course of the non-therapy 4 weeks, while psychotropic drug use gradually increased. This pilot study demonstrates a correlation between lavender oil and improved behavioral and psychological symptoms in patients with FTD. The limited sample size and lack of a control group are limitations to the study; however, the findings are suggestive of the previously stated hypothesis, thus creating a need for further research.

Irmak Sapmaz et al. investigated the efficacy of lavender oil in the treatment of pain. The study was a qualitative double blind, randomized study which included 100 participants diagnosed with renal colic that were placed in 2 groups [23]. Pain was evaluated in each patient utilizing the Visual Analogue Scale. Data was then recorded utilizing the Levene test and chi-squared test; statistical analysis was done via IMB SPSS 20.0 software [23]. Demographic characteristics included fifty-nine men, forty-one women and a mean age for both groups between ages 36 and 38. The study concluded there to be a correlation between pain relief and lavender oil when given in conjunction with conventional therapy, such as NSAIDs, within 30 minutes [23]. This study is another example of the how aromatherapy potentially affects the body physiologically and psychologically in regards to discomfort and body imbalance.

Schneider, conducted a study which investigated the efficacy of an
Aromastick on stress. The sample size included 90 participants and inclusion criteria consisted of: legal age, no current medications, a history of elevated stress in the past 30 days, no diabetes, no heart disease and no mental illness. Qualifying participants were given two standardized questionnaires: The Chronic Stress and Burnout Scale (CSBS) and the Mood and Wellbeing Checklist (MWC). The control group was given a placebo inhaler and one experimental group was given an aromastick inhaler and the other experimental group was exposed to scented air. All groups participated in progressive muscle relaxation during the study. Each individual was tested separately and a baseline heart rate, blood pressure and mood were obtained before the therapy. The control group did not vary with mood or physiologically. The physiological effects of the aromatherapy were realized during the study with the two experimental groups; the heart rate and blood pressure dropped dramatically with aromatherapy [24].

Cino conducted a prospective, randomized control study to compare the efficacy of aromatherapy and hand massage to non-aromatherapy hand massage on individuals living in long term care with chronic pain. The study included 7 nursing homes located in the mid-Atlantic of the United States. Qualifying participants included 118 individuals living in a long term care facility for at least 3 months. Inclusion critera included age 60 and older with chronic pain and Mental Status score of 8 out of 15 or more. Exclusion criteria included open wounds, those with skin disease and sensory loss of the lower arms [24]. Two groups were formulated, with one group receiving the $\mathrm{M}$ technique hand massage with lavender oil and one group receiving just the $\mathrm{M}$ technique hand massage without aromatherapy. The Geriatric Multidimensional Pain Illness Inventory tool was used to evaluate pain level in the individuals and evaluated 3 dimensions: pain and suffering, life interference and emotional distress in longterm care residents. Data was then analyzed utilizing the SPSS coding system. The study findings found no difference in therapeutic effect with either group. Limitations to the study were that $98 \%$ of the participants were on some sort of pain medication regimen, the clinical fact that sense of smell diminishes with advancing age and inconsistency with participation with the participants [25].

Johnson et. Al, conducted a study to evaluate the efficiency of aromatherapy with nausea, anxiety and pain in a nurse-driven setting. The qualitative study was conducted in Minneapolis and included 10 hospitals. Over 18,000 qualifying individuals were age 18 and older and included individuals who were receiving nurse-delivered aromatherapy between February 2012 and June 2014. The data was obtained via self-reporting from the patients. Variables such as age, race, marital status, sex were documented and then each patient was categorized based on severity of illness: minor, moderate, major and extreme, respectively. The study concluded there to be a correlation between aromatherapy and decreased symptoms of pain, nausea and anxiety [26]. Limitations to the study were that it was a not a randomized control trial and findings were subjective based on self-reporting by the patients. This study does however, provides evidence of the potential effectiveness of aromatherapy and encourages further studies.

\section{Synthesis of Literature}

Finding information regarding the safety issues with use of psychotropic drugs within the past 10 years was limited. However, research with aromatherapy appears to be on the rise. Psychoactive medications have physical and cognitive sedative effects on individuals, which in turn increases the risk for a fall. Especially in 
the elderly, falls increase the risk for fractures. The risk of mortality related to fractures is high with the elderly population.

Conversely, aromatherapy counteracts with the effects of the sympathetic nervous system by decreasing heart rate, decreasing blood pressure but does not cognitively sedate the individual. Aromatherapy physiologically calms the body, suppresses the body's adversely effecting defense mechanisms such as hives and pain response promoting harmony and balance within the body. Incidentally, although limited, studies have revealed a correlation between the scent of lavender and decreased agitation. More research regarding aromatherapy and its effects on dementia related behaviors specifically is needed.

\section{Implications for Further Research}

There are many side effects and safety concerns with psychotropic medications, especially with the elderly. The fall risk is greatly increased which correlates with fracture risk and mortality risk. More specific research regarding the potential safety risks which arise with the use of psychotropic medications needs to be explored in order to qualify the continued use. Aromatherapy is a safe alternative which has no potential side effects. Moreover, studies have proven there to be a physiological calming/relaxing effect with the use of aromatherapy. There is a need for more recent high quality research regarding the effects of aromatherapy on dementia related behaviors such as randomized control trials with larger samples. Each study should qualify behaviors specifically and test different aspects of aromatherapy individually, such as topical verses candles or sprays etc. Research is scarce in regards to aromatherapy and dementia; however, current research supports the hypothesis that aromatherapy can be an effective intervention for dementia related behaviors and should be further explored.

\section{Conclusion}

The elderly population is growing very rapidly and along with it is the risk for potential aging disease processes which develop later on as a result of the aging process. The need for services to provide for the aging population by proxy will also increase. Quality of care delivered is essential in health care and can only be realized through rigorous appraisal with the support of evidenced based practice. This is the very foundation which determines the efficacy of health care services.

\section{References}

1. CDC (2003) Public Health and Aging: Trends in Aging - United States and Worldwide.

2. WHO (2016) Older Adults | Healthy People 2020.

3. Andrews J (2014) why we need dementia training. Nursing Standard 28: 63.

4. Beck C K, Vogelpohl T S, Rasin J H, Uriri J T, Walls R, et al. (2002) Effects of Behavioral Interventions on Disruptive Behavior and Affect in Demented Nursing Home Residents. Nursing Research 51: 219-228.

5. Maluso-Bolton Tina MN (2000) Terminal Agitation. Journal of Hospice \& Palliative Nursing 2: 21-22.

6. Kohl, Grassley, Blumenthal (2012) Text of S. 3604 (112th): Improving dementia care treatment for older adults act of 2012.

7. Alzheimer's Advocate Center. (2015). Advocacy |Alzheimer's Association. Retrieved from

8. Fox Aisen C (2015) Regenstrief, IU study: Seniors with dementia make more emergency department.
9. Friedman E M, Shih R A, Langa K M, Hurd M D (2015) US prevalence and predictors of informal caregiving for dementia. Health Affairs 34: 1637-1641.

10. Pollard T C (2015) Aroma AcuPoint Therapy: A New, NonInvasive Healing Modality Perfect for Children. Journal Of The American Herbalists Guild 13: 37-44.

11. Algase D L, Beck C, Kolanowski A, Whall A, Berent S, Richards K, Beattie E (1996) Need driven-dementia-compromised behavior: An alternative view of disruptive behavior. American Journal of Alzheimer's disease 11: 10-19.

12. Burns N, Grove S K, Gray J (2015) Understanding nursing research: Building an evidence-based practice (6th ed.). St. Louis, MI: Elsevier Saunders.

13. Chamine I, Oken B S (2016) Aroma Effects on Physiologic and Cognitive Function Following Acute Stress: A Mechanism Investigation. Journal of Alternative \& Complementary Medicine 22: 713-721.

14. Testad I, Auer S, Mittelman M, Ballard C, Fossey J, et al. (2010) Nursing home structure and association with agitation and use of psychotropic drugs in nursing home residents in three countries: Norway, Austria and England. International Journal Of Geriatric Psychiatry 25: 725-731.

15. Yang M, Lin L, Wu S, Chiu J, Wang P, Lin J, London W M (2015) A non-rigorous investigation of aroma-acupressure and aromatherapy treatment of dementia-associated agitation. Focus On Alternative \& Complementary Therapies 20: 146-147.

16. Nobay F, Simon B, Levitt M, Dresden G (2004) A prospective, double-blind, randomized trial of midazolam versus haloperidol versus lorazepam in the chemical restraint of violent and severely agitated patients. Academic Emergency Medicine 11: 744-749.

17. Cürcani M, Tan M (2014) the effect of aromatherapy on haemodialysis patients' pruritus. Journal of Clinical Nursing 23: 3356-3365.

18. Pelletier I C, Landreville P (2007). Discomfort and agitation in older adults with dementia. BMC Geriatrics 7: 27.

19. Ou M, Lee Y, Li C, Wu S (2014) The Effectiveness of Essential Oils for Patients with Neck Pain: A Randomized Controlled Study. Journal of Alternative \& Complementary Medicine 20: 771-779.

20. Finkle W D, Der J S, Greenland S, Adams J L, Ridgeway G, et al. (2011) Risk of Fractures Requiring Hospitalization After an Initial Prescription for Zolpidem, Alprazolam, Lorazepam, or Diazepam in Older Adults. Journal Of The American Geriatrics Society 59:1883-1890.

21. Kirkland L, Kashiwagi D, Burton M, Cha S, Varkey P (2011) The charlson comorbidity index score as a predictor of 30-day mortality after hip fracture surgery. American Journal of Medical Quality 26: 461-467.

22. Kimura T, Takamatsu J (2013) Pilot study of pharmacological treatment for front temporal lobar degeneration: Effect of lavender aroma therapy on behavioral and psychological symptoms. Geriatrics \& Gerontology International 13: 516-517.

23. Irmak Sapmaz H, Uysal M, Taş U, Esen M, Barut M, et al. (2015) The Effect of Lavender Oil in Patients with Renal Colic: A Prospective Controlled Study Using Objective and Subjective Outcome Measurements. Journal of Alternative \& Complementary Medicine 21: 617-622.

24. Schneider R (2016) There Is Something in the Air: Testing the Efficacy of a new Olfactory Stress Relief Method (AromaStick ${ }^{\circledR}$ ). Stress \& Health: Journal of the International Society for the Investigation Of Stress 32: 411-426.

25. Cino K (2014) Aromatherapy Hand Massage for Older Adults 
With Chronic Pain Living in Long-Term Care. Journal of Holistic Nursing 32: 304-313.

26. Johnson J R, Rivard R L, Griffin K H, Kolste A K, Joswiak D, et al. (2016) The effectiveness of nurse-delivered aromatherapy in an acute care setting. Complementary Therapies in Medicine 25: 164-169.

Citation: Vanessa Veit (2018). Aromatherapy Versus Pharmaceutical Interventions for Dementia Related Behaviors Translating \& Integrating Scholarship Practicum. Journal of Medical \& Clinical Research 3(1):1-6.

Copyright: (C2018 Vanessa Veit. This is an open-access article distributed under the terms of the Creative Commons Attribution License, which permits unrestricted use, distribution, and reproduction in any medium, provided the original author and source are credited. 